Department of Engineering Mechanics, Hetman Petro Sahaidachnyi National Army Academy, 32, Heroes of Maidan Str., Lviv, Ukraine, E-mail: vrublew@ gmail.com

\title{
PROSPECTS OF USE OF VIBRATORY DEVICES WITH ELECTROMAGNETIC DRIVES FOR MASSIVE PIECE GOODS CONVEYING
}

Received: November 10, 2017 / Revised: December 20, 2017 / Accepted: December 26, 2017

(C) Vrublevskyi I., 2017

\begin{abstract}
Vibratory conveying devices with electromagnetic drive are widely used in the different branches of industry. As a rule, they deal with relatively small piece goods and are not suitable for conveying of the large massive goods with high productivity. The conveying of massive piece goods needs the using of non-hopping modes of moving in which the high velocity can be reached only in the case of non-linear vibration trajectories of the conveying track, for example elliptical trajectories. Such trajectories are obtained by using of the independent drives of horizontal and vertical oscillations. Even greater velocity can be reached by using biharmonic vertical oscillation with particular optimal relations between amplitudes and phase differences of the component oscillations. These types of devices can be designed on a basis of three-mass oscillating system.
\end{abstract}

Theoretical and experimental investigations show that the goods' mass change significantly affects the vertical oscillations and weakly affects the horizontal oscillations. And at the certain relations between masses ratio and resonant tuning (ratio of frequencies of forced and natural oscillations) the vertical oscillations of active mass of two-mass vibratory device are insensitive to the change of goods' mass. The sensitivity of vibratory conveying stability to the goods' mass change was investigated for two-mass vibratory devices with elliptical oscillations. Three-mass oscillating systems with biharmonic oscillations in the point of view of the sensitivity to the goods' mass changing were not considered.

Analysis of different three-mass oscillating systems with electromagnetic drives of harmonic horizontal and biharmonic vertical oscillations allows determining two most preferable versions. The equations for amplitudes of components and frequencies of natural oscillations determining are derived. From the condition of insensitivity to conveying mass change the optimal relations between masses ratio and resonant tuning are obtained.

Keywords: vibratory conveying; electromagnetic drive; biharmonic oscillations; three-mass oscillating system.

\section{Introduction}

Vibratory conveying devices with electromagnetic drives (vibratory bowl feeders, through and spiral conveyors, vibratory manipulators) are widely used for conveying, sieving, dosing and feeding of bulk and piece goods in the different branches of industry [1]. They are easy to maintain and are reliable for exploitation due to the absence of rubbing parts and details except the conveying track, they are economical thanks to the resonant modes of drive's work. But as a rule such devices with electromagnetic drives deal with relatively small piece goods with light masses. The existing constructions of vibratory devices are not suitable for conveying of the large massive goods with high productivity because with increasing of goods' weight the conveying velocity dramatically decreases until complete impossibility of goods' moving. This disadvantage may be eliminated in new constructions of vibratory devices. 


\section{Problem Statement}

The vibratory conveying of the massive goods with high productivity needs the using of nonhopping modes of moving and the high conveying velocity. But the high velocity in non-hopping modes can be reached only in the case of non-linear vibration trajectories of the conveying track. Such trajectories are obtained by using of the independent drives of harmonic horizontal and vertical oscillations with the phase difference between them when the conveying track of device oscillates along elliptical trajectory. Even greater conveying velocity can be reached by using of biharmonic vertical oscillations with the particular optimal relations between amplitudes and phase differences of the component oscillations.

These types of relatively complex oscillations can be obtained in the vibratory devices designed on a basis of a three-mass oscillating system. One of the serious problems of conveying of the massive goods is the sensitivity of its stability to their mass change. A significant decreasing of this sensitivity can be achieved in the vibratory devices by determining the optimal ratio of masses and frequencies of natural oscillations. That's why the design and investigations of new constructions of vibratory devices with electromagnetic drives suitable for conveying of the massive goods with high productivity is actual problem.

\section{Review of Modern Information Sources on the Subject of the Paper}

Vibratory conveying process was considered within the framework of theory of massive point particle moving along a declined plane in [1,2]. Experimental research of the vibratory conveying of piece goods in non-hopping modes of moving proves the validity of this theory $[3,4]$. The use of elliptical and biharmonic oscillations allows increasing the conveying velocity and the track's inclination angle and provides the possibility to reverse the details moving. The sensitivity of vibratory conveying stability to the goods' mass change were considered in [5] for two-mass vibratory devices with elliptical oscillations. Vibratory devices designed on the basis of three-mass oscillating system with two resilient systems research in [6] but there were considered only devices with linear vibration trajectories. Three-mass oscillating systems with biharmonic oscillations in the point of view of the sensitivity to the goods' mass changing were not considered.

\section{Objectives and Problems of Research}

The purpose of research is the design and investigation of construction of three-mass vibratory conveying device with independent electromagnetic drives of horizontal harmonic and vertical biharmonic oscillations that is insensitive to changing of conveying piece goods' mass.

\section{Main Material Presentation}

The conveying velocity $V$ can be calculated by formula [5]:

$$
V=A_{x} \cdot \omega \cdot K_{V}
$$

where $A_{x}$ is the amplitude of horizontal oscillations; $\omega$ is the frequency of forced oscillations; $K_{V}$ is the dimensionless coefficient of velocity, depending in non-hopping modes of moving on the next dimensionless parameters: $K_{\alpha}$ is the track angle inclination parameter, $K_{\beta}$ is the vibration angle parameter, $w$ is the parameter of overload, and

$$
K_{\alpha}=\frac{\tan \alpha}{f} ; \quad \quad K_{\beta}=\frac{A_{x}}{A_{z} \cdot f} ; \quad w=\frac{A_{z} \omega^{2}}{g \cos \alpha},
$$

where $\alpha$ is the track angle inclination; $f$ is the coefficient of friction; $g$ is the acceleration of gravity; $A_{z}$ is the amplitude of vertical oscillations. The increase of amplitude $A_{x}$ and frequency $\omega$ of oscillations is limited by the constructive parameters of conveying device, that's why the maximal value of $K_{V}$ ensuring is very important.

The disadvantage of vibratory conveying with linear vibrations is the decrease of coefficient of velocity $K_{V}$ with increasing of vibration angle parameter $K_{\beta}$. Thus at large amplitudes $A_{x} \gg A_{z}$, the 
value of $K_{V}$ is small and the conveying is ineffective. The essential property of vibratory conveying with independent horizontal and vertical oscillations with optimal parameters (particularly elliptical vibrations) is that coefficient of velocity $K_{V}$ almost doesn't depend on vibration angle parameter $K_{\beta}$ [4]. In nonhopping modes of moving coefficient of velocity $K_{V}$ is maximal at $w=1$. Then with optimal ratio of amplitudes and phase differences of component oscillations the coefficient of velocity $K_{V}$ depends only on track angle inclination parameter $K_{\alpha}$. The coefficient of velocity $K_{V}$ dependence on the track angle inclination parameter $K_{\alpha}$ for elliptical vibration is shown in Fig. 1 (curve 1).

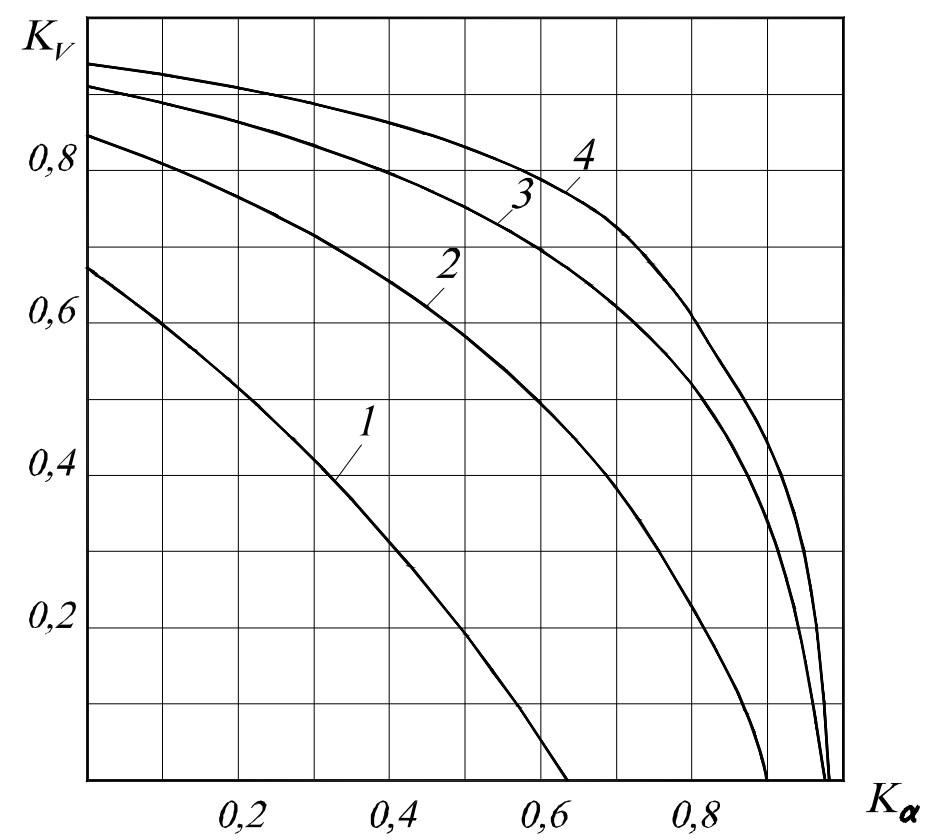

Fig. 1. The coefficient of velocity $K_{V}$ dependence on the track angle inclination parameter $K_{\alpha}$ with the parameter of overload $w=1$ and different numbers of harmonics of vertical oscillations

The large amplitude $A_{x}$ provides the high conveying velocity, that's why electromagnetic drive of horizontal oscillations must work in near-resonant mode, and horizontal oscillations must be harmonic. But amplitude $A_{z}$ of vertical oscillations must be small to provide non-hopping modes of conveying, and electromagnetic drive of vertical oscillations may work far from resonance, where oscillations are not sinusoidal. The researching [4], [7] show that polyharmonic vertical oscillations with optimal parameters allow to increase the value of coefficient of velocity $K_{V}$ in comparison with elliptical oscillations.

And horizontal oscillations (in the direction of conveying - axis $x$ ) and vertical oscillations (in the direction perpendicular to the plane of conveying - axis $y$ ) must be varied under the law:

$$
\begin{gathered}
x=A_{x} \cdot \sin \left(\omega t+\varepsilon_{0}\right) ; \\
y=\sum_{i=1}^{n} A_{i} \cdot \sin \left(i \omega t+\frac{\pi}{2}(i-1)\right),
\end{gathered}
$$

where $n$ is the number of harmonics of polyharmonic vertical oscillations; $A_{i}$ is the amplitude of $i^{\text {th }}$ harmonic; $\varepsilon_{0}$ is the optimal phase difference angle $\left(0 \leq \varepsilon_{0}<\pi / 2\right)$ [8]; $\omega$ is the frequency of forced oscillations; $t$ is time; maximal amplitude of vertical oscillations $A_{z}=\sum_{i=1}^{n} \stackrel{r}{A_{i}}$. 
Fig. 1 shows the coefficient of velocity $K_{V}$ dependence on the track angle inclination parameter $K_{\alpha}$ for vibratory conveying with polyharmonic vertical oscillations with different numbers of harmonics in non-hopping modes $\left(w=1, K_{\beta}>10-20\right)$. As it seen in Fig. 1 biharmonic vertical oscillations $(n=2)$ provide the essential increase of the coefficient of velocity $K_{V}$ in comparison with elliptical oscillations $(n=1)$ especially with large inclination angles. In addition, with every next harmonic $(n=3,4)$ of vertical oscillations the increment of velocity increasing becomes smaller. Moreover, the realization of polyharmonic oscillations with a large number of harmonics is a complex problem. That is why the most preferred use in vibratory conveying device for massive goods with high productivity is biharmonic vertical oscillations of conveying track.

Such devices can be created as a three-mass oscillating system. A comparative analysis of different versions of dynamic schemes of three-mass oscillating system of vibratory conveying device with electromagnetic drives of biharmonic vertical oscillations and harmonic horizontal oscillations [4] made it possible to determine two most preferable versions that are shown in Fig. 2.

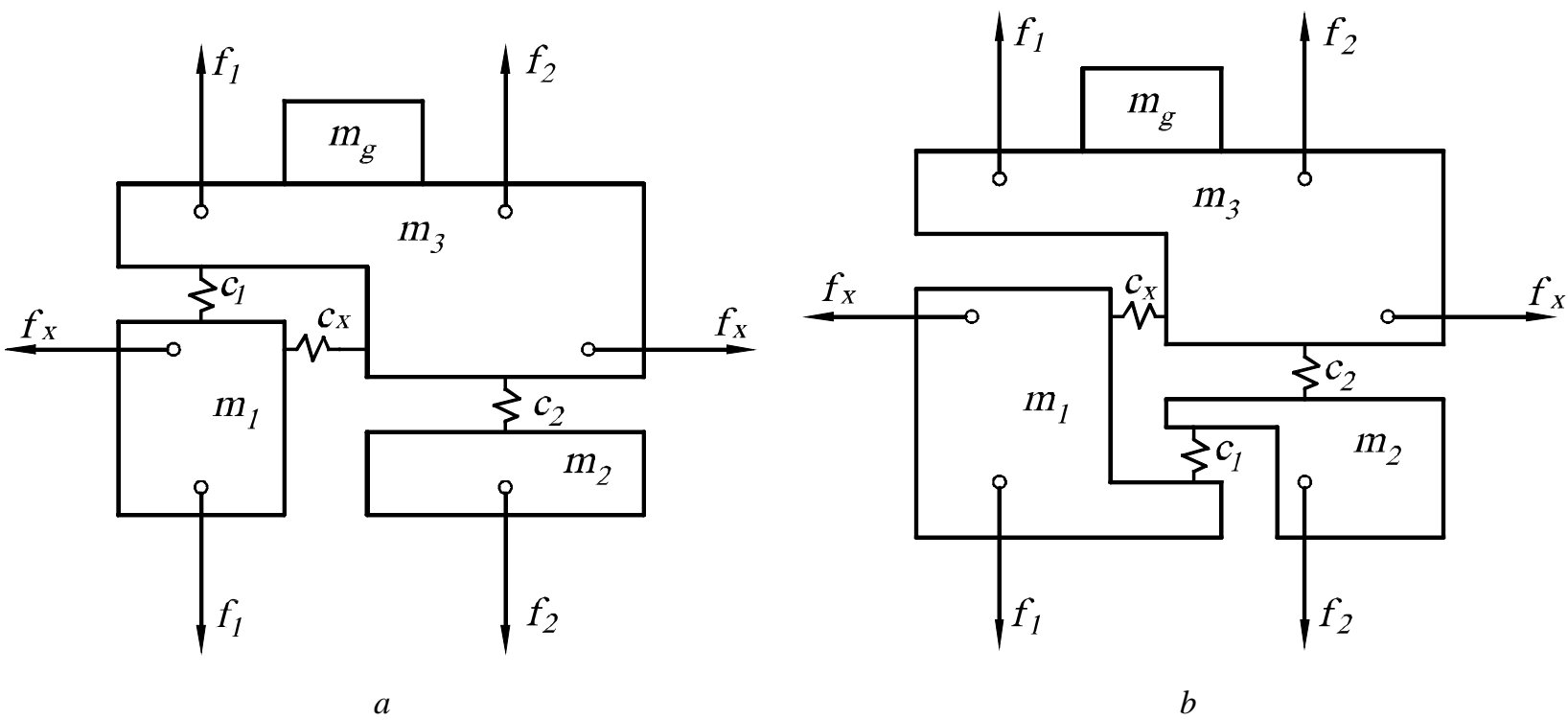

Fig. 2. Dynamic schemes of three-mass vibratory conveying devices with electromagnetic drives of horizontal harmonic and vertical biharmonic oscillations

The system of horizontal oscillations is actually a two-mass system in both versions. Active mass $m_{3}$ with conveying track, on which piece goods with mass $m_{g}$ move, and intermediate mass $m_{2}$ oscillate horizontally as a whole. They are connected with reactive mass $m_{1}$ by resilient system with stiffness $c_{x}$. The electromagnetic drive of horizontal oscillations with effort $f_{x}$ is located between reactive mass $m_{1}$ and active mass $m_{3}$. Three-mass systems of vertical oscillations in both versions are different. In first version (Fig. 1,a) reactive mass $m_{1}$ and active mass $m_{3}$, between which electromagnetic drive of first harmonic of vertical oscillations with effort $f_{1}$ is placed, are connected by resilient system with stiffness $c_{1}$. The electromagnetic drive of second harmonic of vertical oscillations with effort $f_{2}$ is located between active mass $m_{3}$ and intermediate mass $m_{2}$ that are connected by resilient system with stiffness $c_{2}$. In the second version (Fig. 2, $b$ ) the locations of electromagnetic drives are the same. But resilient system with stiffness $c_{1}$ connects reactive mass $m_{1}$ and intermediate mass $m_{2}$. And resilient system with stiffness $c_{2}$ connects active mass $m_{3}$ and intermediate mass $m_{2}$. 
The efforts of electromagnetic exciters varies under the next law:

$$
f_{1}=F_{1} \cos \omega t ; \quad f_{2}=F_{2} \cos 2(\omega t+\varphi),
$$

where $F_{1}$ and $F_{2}$ are the amplitudes of disturbing forces; $\varphi$ is the phase difference angle.

Theoretical and experimental investigations show that the goods' mass changing significantly affects the vertical oscillations and weakly affects the horizontal oscillations [5]. In addition, at the certain relations between masses ratio and resonant tuning the vertical oscillations of active mass of two-mass vibratory device are insensitive to the change of goods' mass.

For the first version of three-mass device (Fig. 1,a) the law of the active mass vertical oscillations can be represented as follow [9]:

where

$$
y_{3}=\frac{F_{1}\left(\omega^{2}-c_{2} / m_{2}\right)}{\Delta_{1}} \cos \omega t-\frac{F_{2}\left(4 \omega^{2}-c_{1} / m_{1}\right)}{\Delta_{2}} \cos 2(\omega t+\varphi),
$$

$$
\begin{gathered}
\Delta_{1}=\left(m_{3}+m_{g}\right)\left\{\omega^{4}-\left[\frac{c_{1}\left(m_{1}+m_{3}+m_{g}\right)}{m_{1}\left(m_{3}+m_{g}\right)}+\frac{c_{2}\left(m_{2}+m_{3}+m_{g}\right)}{m_{2}\left(m_{3}+m_{g}\right)}\right] \omega^{2}+c_{1} c_{2} \frac{m_{1}+m_{2}+m_{3}+m_{g}}{m_{1} m_{2}\left(m_{3}+m_{g}\right)}\right\} ; \\
\Delta_{2}=\left(m_{3}+m_{g}\right)\left\{16 \omega^{4}-4\left[\frac{c_{1}\left(m_{1}+m_{3}+m_{g}\right)}{m_{1}\left(m_{3}+m_{g}\right)}+\frac{c_{2}\left(m_{2}+m_{3}+m_{g}\right)}{m_{2}\left(m_{3}+m_{g}\right)}\right] \omega^{2}+c_{1} c_{2} \frac{m_{1}+m_{2}+m_{3}+m_{g}}{m_{1} m_{2}\left(m_{3}+m_{g}\right)}\right\} .
\end{gathered}
$$

As the value of goods' mass $m_{g}$ is contained in this equation only in $\Delta_{1}$ and $\Delta_{2}$ it is sufficient for stabilization of $y_{3}$ to stabilize $\Delta_{1}$ and $\Delta_{2}$. Let's introduce the next quantities: $n_{1}, n_{2}, n_{0}$ are the ratios of masses; $p_{1}$ and $p_{2}$ are the partial frequencies; $\rho_{1}$ and $\rho_{2}$ are the partial tunings: $n_{1}=\frac{m_{1}}{m_{3}} ; n_{2}=\frac{m_{2}}{m_{3}}$; $n_{0}=\frac{m_{g}}{m_{3}} ; p_{1}^{2}=\frac{c_{1}\left(m_{1}+m_{2}\right)}{m_{1} m_{2}} ; p_{2}^{2}=\frac{c_{2}\left(m_{2}+m_{3}\right)}{m_{2} m_{3}} ; \rho_{1}=\frac{p_{1}}{\omega} ; \rho_{2}=\frac{p_{2}}{\omega}$.

Then let's transform equations (4):

$$
\begin{gathered}
\Delta_{1}=m_{3} \omega^{2}\left\{1-\rho_{1}^{2}-\rho_{2}^{2}+\frac{\rho_{1}^{2} \rho_{2}^{2}\left(1+n_{1}+n_{2}\right)}{\left(1+n_{1}\right)\left(1+n_{2}\right)}+n_{0}\left[1-\frac{\rho_{1}^{2}}{1+n_{1}}-\frac{\rho_{2}^{2}}{1+n_{2}}+\frac{\rho_{1}^{2} \rho_{2}^{2}}{\left(1+n_{1}\right)\left(1+n_{2}\right)}\right]\right\} \\
\Delta_{2}=m_{3} \omega^{2}\left\{16-4\left(\rho_{1}^{2}+\rho_{2}^{2}\right)+\frac{\rho_{1}^{2} \rho_{2}^{2}\left(1+n_{1}+n_{2}\right)}{\left(1+n_{1}\right)\left(1+n_{2}\right)}+n_{0}\left[16-\frac{4 \rho_{1}^{2}}{1+n_{1}}-\frac{4 \rho_{2}^{2}}{1+n_{2}}+\frac{\rho_{1}^{2} \rho_{2}^{2}}{\left(1+n_{1}\right)\left(1+n_{2}\right)}\right]\right\} .
\end{gathered}
$$

To stabilize $\Delta_{1}$ and $\Delta_{2}$ it is sufficient that expressions in square brackets for $n_{0}$ in equations (5) and (6) be zero:

$$
\begin{gathered}
1-\frac{\rho_{1}^{2}}{1+n_{1}}-\frac{\rho_{2}^{2}}{1+n_{2}}+\frac{\rho_{1}^{2} \rho_{2}^{2}}{\left(1+n_{1}\right)\left(1+n_{2}\right)}=0 \\
16-\frac{4 \rho_{1}^{2}}{1+n_{1}}-\frac{4 \rho_{2}^{2}}{1+n_{2}}+\frac{\rho_{1}^{2} \rho_{2}^{2}}{\left(1+n_{1}\right)\left(1+n_{2}\right)}=0 .
\end{gathered}
$$

System of equations (7) reduces to two unbound equations:

$$
1+n_{1}=\rho_{1}^{2} ; \quad 1+n_{2}=\rho_{2}^{2} / 4 .
$$

The condition of insensitivity of harmonic vertical oscillations (elliptical vibrations) in two-mass vibratory conveying device can be regarded as a particular case when $n_{2}=0$ and $\rho_{2}=0$. And this condition reduces to the first equation (8). The results of calculations of this equation show the coincidence with the graphs' data obtained in [5].

The frequencies of natural oscillations are expressed in terms of the partial frequencies as follows:

$$
\omega_{1,2}{ }^{2}=0,5\left[p_{1}^{2}+p_{2}^{2} \mathrm{~m} \sqrt{\left(p_{1}^{2}-p_{2}{ }^{2}\right)^{2}+\frac{4 p_{1}^{2} p_{2}^{2} n_{1} n_{2}}{\left(1+n_{1}\right)\left(1+n_{2}\right)}}\right] .
$$


Expressing $p_{1}$ and $p_{2}$ through $n_{1}$ and $n_{2}$ according to the formula (8) and substituting in (9), we obtain:

$$
\omega_{1,2}{ }^{2}=0,5 \omega^{2}\left[5+n_{1}+4 n_{2} \mathrm{~m} \sqrt{\left(n_{1}-4 n_{2}-3\right)^{2}+16 n_{1} n_{2}}\right] .
$$

For biharmonic oscillations the optimal ratio of frequencies of natural oscillations is $\omega_{2} / \omega_{1}=2$, then given by the value of the resonance tuning at the first natural frequency $z=\omega / \omega_{1}$, we can find the values of optimal ratios of masses $n_{1}=\frac{4 z^{-4}-5 z^{-2}+1}{3} ; n_{2}=\frac{5 z^{-2}-z^{-4}-4}{3}$.

The graphs of optimal masses ratio $n_{1,2}$ and partial frequencies $p_{1,2}$ dependences on resonant tuning $z$ are shown in Fig. 3, $a$. The graphs of optimal masses dependences on resonant tuning $z$ are represented by continuous lines, the graphs of ratio of partial frequencies $p_{1,2}$ to frequency of force oscillations $\omega$ on resonant tuning $z$ are represented by dashed lines.

Now let us consider the second version of three-mass oscillating system (Fig. 2, b) by analogy with the first version. The law of the active mass vertical oscillations:

$$
y_{3}=\frac{F_{1}\left(\omega^{2}-\frac{c_{1}\left(m_{1}+m_{2}\right)}{m_{1} m_{2}}-\frac{c_{2}}{m_{2}}\right)}{\Delta_{1}} \cos \omega t-\frac{F_{2}\left(4 \omega^{2}-\frac{c_{1}\left(m_{1}+m_{2}\right)}{m_{1} m_{2}}\right)}{\Delta_{2}} \cos 2(\omega t+\varphi),
$$

where

$$
\begin{gathered}
\Delta_{1}=\left(m_{3}+m_{g}\right)\left\{\omega^{4}-\left[\frac{c_{1}\left(m_{1}+m_{2}\right)}{m_{1} m_{2}}+\frac{c_{2}\left(m_{2}+m_{3}+m_{g}\right)}{m_{2}\left(m_{3}+m_{g}\right)}\right] \omega^{2}+c_{1} c_{2} \frac{m_{1}+m_{2}+m_{3}+m_{g}}{m_{1} m_{2}\left(m_{3}+m_{g}\right)}\right\} ; \\
\Delta_{2}=\left(m_{3}+m_{g}\right)\left\{16 \omega^{4}-4\left[\frac{c_{1}\left(m_{1}+m_{2}\right)}{m_{1} m_{2}}+\frac{c_{2}\left(m_{2}+m_{3}+m_{g}\right)}{m_{2}\left(m_{3}+m_{g}\right)}\right] \omega^{2}+c_{1} c_{2} \frac{m_{1}+m_{2}+m_{3}+m_{g}}{m_{1} m_{2}\left(m_{3}+m_{g}\right)}\right\} .
\end{gathered}
$$

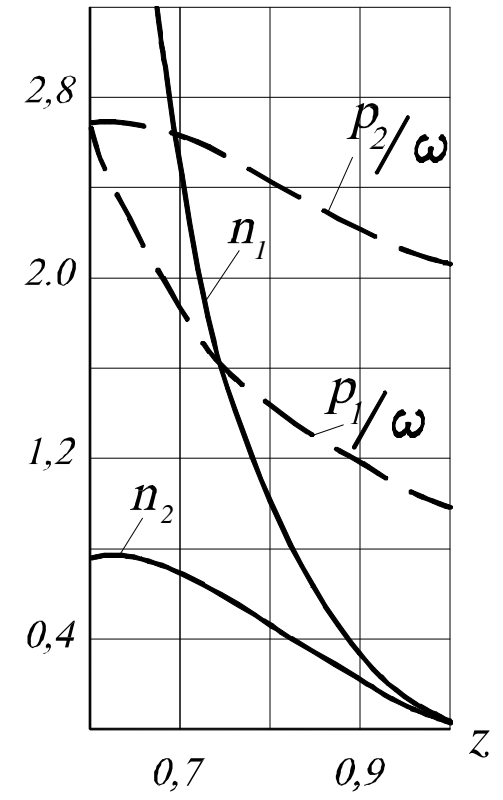

$a$

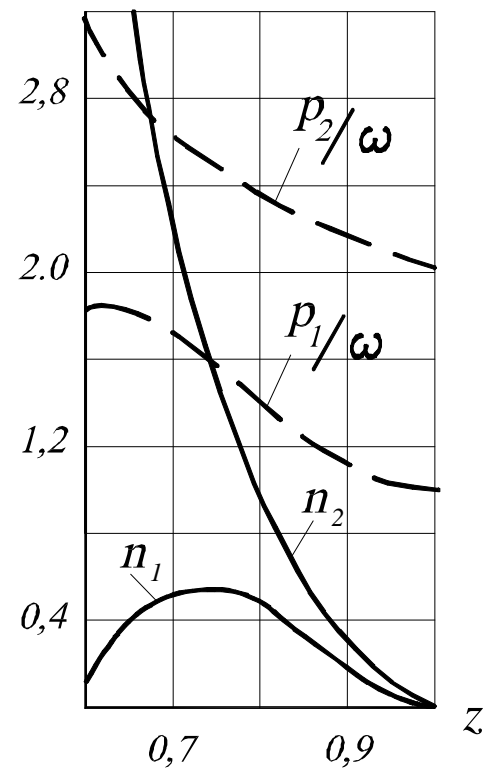

$b$

Fig. 3. The graphs of optimal masses ratio $n_{1,2}$ and partial frequencies $p_{1,2}$ dependences on resonant tuning $z$

By analogy with equations (5) and (6), for first version of three-mass system we obtain:

$$
\Delta_{1}=m_{3} \omega^{2}\left\{1-\rho_{1}^{2}-\rho_{2}^{2}+\frac{\rho_{1}^{2} \rho_{2}^{2}\left(1+n_{1} n_{2}+n_{2}\right)}{\left(1+n_{1}\right)\left(1+n_{2}\right)}+n_{0}\left[1-\rho_{1}^{2}-\frac{\rho_{2}^{2}}{1+n_{2}}+\frac{\rho_{1}^{2} \rho_{2}^{2}}{\left(1+n_{1}\right)\left(1+n_{2}\right)}\right]\right\} ;
$$




$$
\Delta_{2}=m_{3} \omega^{4}\left\{16-4\left(\rho_{1}^{2}+\rho_{2}^{2}\right)+\frac{\rho_{1}^{2} \rho_{2}^{2}\left(1+n_{1} n_{2}+n_{2}\right)}{\left(1+n_{1}\right)\left(1+n_{2}\right)}+n_{0}\left[16-4\left(\rho_{1}^{2}+\frac{\rho_{2}^{2}}{1+n_{2}}\right)+\frac{\rho_{1}^{2} \rho_{2}^{2}}{\left(1+n_{1}\right)\left(1+n_{2}\right)}\right]\right\} .
$$

And by analogy the stability conditions are that expressions in square brackets for $n_{0}$ in equations (11) be zero:

$$
\begin{gathered}
1-\rho_{1}^{2}-\frac{\rho_{2}^{2}}{1+n_{2}}+\frac{\rho_{1}^{2} \rho_{2}^{2}}{\left(1+n_{1}\right)\left(1+n_{2}\right)}=0 ; \\
16-4\left(\rho_{1}^{2}+\frac{\rho_{2}^{2}}{1+n_{2}}\right)+\frac{\rho_{1}^{2} \rho_{2}^{2}}{\left(1+n_{1}\right)\left(1+n_{2}\right)}=0 .
\end{gathered}
$$

The system of equations (12) is reduced to the next form:

$$
\rho_{1}^{2}+\frac{\rho_{2}^{2}}{1+n_{1}}=5 ; \quad \frac{\rho_{1}^{2} \rho_{2}^{2}}{\left(1+n_{1}\right)\left(1+n_{2}\right)}=4
$$

And from system (13) we find the values of optimal ratios of masses depending on the partial frequencies:

$$
n_{1}=\frac{\rho_{1}^{2} \rho_{2}^{2}}{4\left(1+n_{2}\right)}-1 ; \quad n_{2}=\frac{\rho_{2}^{2}}{5-\rho_{1}^{2}}-1
$$

A joint solution of systems of equations (10) and (14) will allow calculating the dependences of optimal masses ratios $n_{1,2}$ and $\rho_{1,2}$ on resonant tuning $z$ under condition $\omega_{2} / \omega_{1}=2$. The graphs of these dependences for second version of three-mass vibratory conveying device are shown on Fig. $3, b$.

The comparison of two considered versions of three-mass conveying device with polyharmonic vertical oscillations allows us to conclude that the first version is preferable because in the second version the values of optimal masses ratios are far from the possibility of obtaining the large amplitude $A_{x}$ of horizontal oscillations with minimal energy costs (large values of $n_{2}$ and small values of $n_{1}$ ).

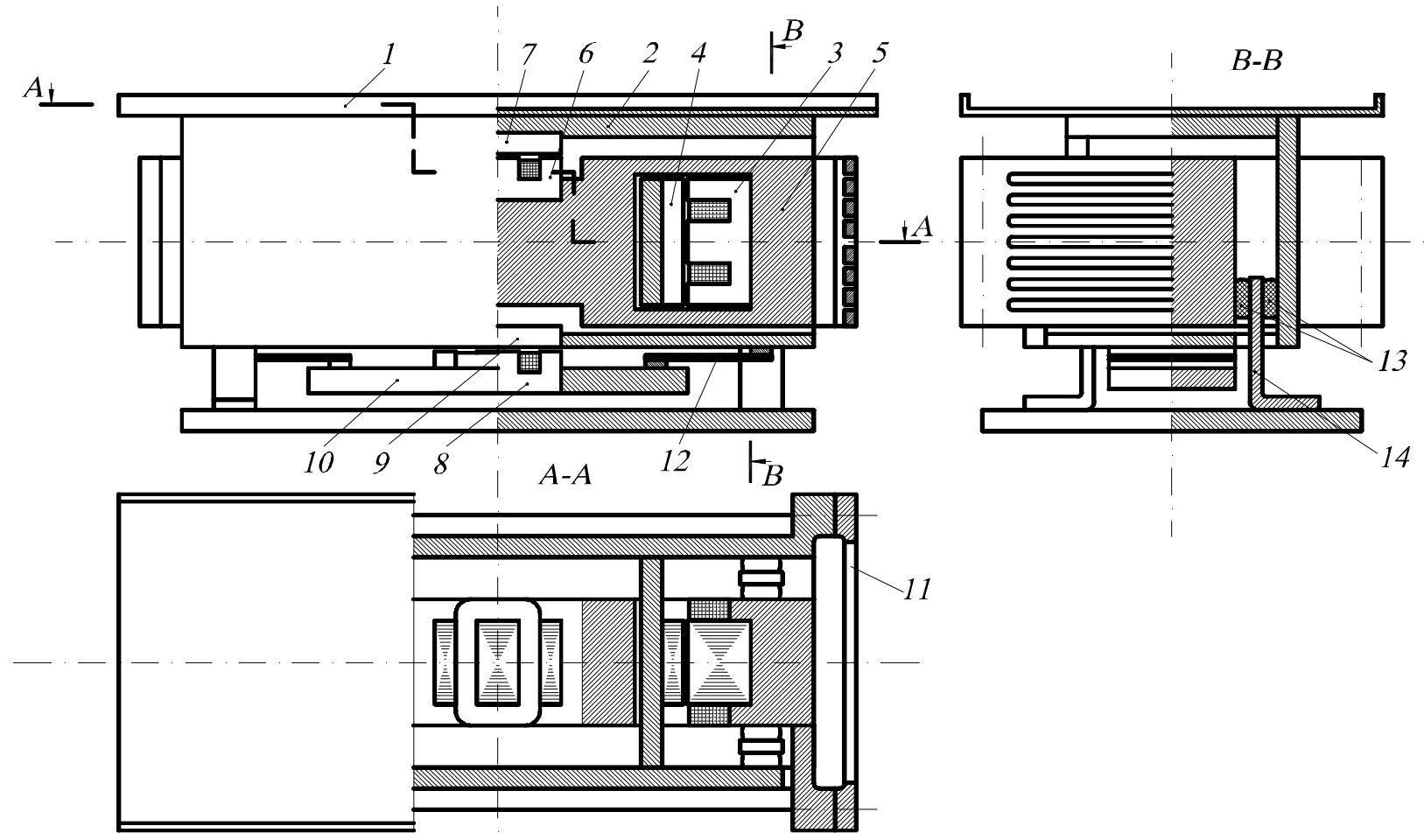

Fig. 4. The construction of three-mass vibratory conveyor with electromagnetic drives of harmonic horizontal and biharmonic vertical oscillations 
The construction of three-mass vibratory conveyor with electromagnetic drives of harmonic horizontal and biharmonic vertical oscillations is shown in Fig. 4 [10]. A main view with frontal slice is in Fig. 4, $a$; a top view with horizontal slice $A-A$ is in Fig. $4, b$; a left view with profile slice $B-B$ is in Fig. $4, c$. Vibratory conveyor consists of three oscillating masses: active, intermediate and reactive. The hatch of active and intermediate masses and the hatch of reactive mass in Fig. 4 are in the different directions. The active mass consists of through conveying track 1, connected to box frame 2 on which the anchors of all electromagnetic exciters are mounted. Electromagnetic drive of vibratory conveyor includes two electromagnetic exciters of horizontal oscillations and two electromagnetic exciters of vertical oscillations. Two identical exciters of horizontal oscillations work in antiphase to obtain greater amplitude. Each of them includes the electromagnet 3 that is mounted on the reactive frame 5 and the anchor 4 . One of the electromagnetic exciters of vertical oscillations excites the force with a frequency equal to a frequency of exciters of horizontal oscillations. Another one excites the force with a frequency twice as this. The first exciter consists of the electromagnet 6 that is mounted on the reactive frame 5 and the anchor 7 . The second exciter consists of the electromagnet 8 that is mounted on the plate 10 and the anchor 9 . Thus, the intermediate mass includes the plate 10 and the electromagnet 8 ; the reactive mass includes the reactive frame 5 and the electromagnets 3 and 6 . The boxed frame 2 is connected with the reactive frame 5 by two latticed leaf springs 11. Such elastic system has suppleness in both horizontal and vertical directions. The boxed frame 2 is connected with the plate 10 by two flat leaf springs 12 . The stiffness of elastic systems according to the notation in Fig. 2:

$$
c_{x}=\frac{2 j E b_{1} h_{1}^{3}}{l_{1}^{3}}, \quad c_{1}=\frac{2 j E b_{1}{ }^{3} h_{1}}{l_{1}^{3}}, \quad c_{2}=\frac{2 E b_{2} h_{2}^{3}}{l_{2}^{3}},
$$

where $E$ is the elastic modulus; $l_{1}$ and $h_{1}$ are the length and the thickness of latticed leaf spring; $j$ and $b_{1}$ are the number and the width of latticed spring's rods, $l_{2}, b_{2}$ and $h_{2}$ are the length, the width and the thickness of the flat leaf spring.

The boxed frame 2 and the reactive frame 5 are separated by vibration isolators 13, between which four brackets 14 are installed and fixed to the foundation. The electromagnetic exciters of vertical oscillations excite biharmonic oscillations, ratio of amplitudes of each harmonic and phase difference angle of which can be regulated to optimal values.

\section{Conclusions}

Vibratory conveying devices with electromagnetic drives of harmonic horizontal and vertical biharmonic oscillations allow reaching a high velocity in non-hopping modes which are necessary for conveying of massive piece goods. Such devices can be created on a basis a three-mass oscillating system. A comparative analysis of different versions of dynamic schemes of three-mass oscillating system of vibratory conveying device was carried out. Equations describing the frequencies of natural oscillations and amplitude of active mass oscillations were derived. The conditions of device's insensitivity to conveying goods' mass change were considered. According these conditions the graphs of mass ratio and ratio of partial frequencies to natural frequency dependence on ratio of frequencies forced and natural oscillations are constructed. After comparison a better version of three-mass oscillation system is chosen from the condition of insensitivity to conveying goods' mass change. The construction of three-mass conveyor based on this choice was developed.

\section{References}

[1] Y. Y. Lavendal, "Vibratsionnyie protsessy i mashiny" ["Vibratory machines and processes"], in Vibratsii v tehnike: Spravochnik [Vibrations in technology: Handbook], V. N. Chelomey, Ed. Moscow, Russia: Mashinostroenie Publ., 1981, vol. 4, 509 p. [in Russian].

[2] I. I. Blehman and G. Ju. Dzhanelidze, Vibracionnoe peremeshchenie [Vibrational movement], Moscow, Russia: Nauka Publ., 1964, 412 p. [in Russian]. 
[3] A. H. Redford and G. Boothroyd, "Vibratory feeding," Proceedings of the Institute of Mechanical Engineering, vol. 182, no. 6, pp. 135-152, 1968.

[4] I. I. Vrublevskij, "Razrabotka i issledovanie vibracionnyh ustrojstv, osushhestvljajushhih organizaciju rabochej sredy robotosistem" ["Development and research of vibration devices that organize the working environment of robotic systems"], Ph. D. dissertation, Lviv Polytechnic National University, Lviv, Ukraine, 1986. [in Russian].

[5] V. O. Povidailo, Vibratsiini protsesy ta obladnannia [Vibratory processes and equipment]. Lviv, Ukraine: Lviv Polytechnic Publishing House, 2004. [in Ukraine].

[6] O. S. Lanets, Vysokoefektyvni mizhrezonansni vibratsiini mashyny z elektromahnitnym pryvodom [Highefficient inter-resonant vibratory machines with electromagnetic drive]. Lviv, Ukraine: Lviv Polytechnic Publishing House, 2008. [in Ukraine].

[7] I. I. Vrublevskyi, "Optymalnyi za shvydkistiu zakon dvokomponentnykh kolyvan vibratsiinykh transportnykh prystroiv z elektromahnitnym pryvodom” ["Optimal by Velocity Two-Component Oscillation Law of Vibratory Conveying Devices with the Electromagnetic Drive”], Viiskovo-tekhnichnyi zbirnyk [Military Technical Collection], vol. 1 (10), pp. 13-16, 2014. [in Ukrainian].

[8] I. Vrublevskyi, "The Phase Difference between Components of Elliptical Oscillations of Vibratory Conveyor Providing Maximum Conveying Velocity," Ukrainian Journal of Mechanical Engineering and Materials Science, vol. 1, no. 1, pp. 47-54, 2015.

[9] I. I. Vrublevskij, "Dinamika trehmassnogo vibrotransportera s bigarmonicheskimi vertikal'nymi kolebanijami" ["Dynamics of a three-mass vibratory transporter with biharmonic vertical oscillations"], Vestnik L'vovskogo politehnicheskogo instituta [Bulletin of Lviv Polytechnic Institute], no. 209, pp. 23-25, 1986. [in Russian].

[10] I. I. Vrublevskij, "Vibracionnyj konvejer” ["Vibratory conveyer”], USSR Patent № 1320135, June 30, 1987. [In Russian]. 